\title{
Regionalization of Bangladesh: A Key to Identify Regional Disparities of the Pattern of Population Distribution
}

\author{
Aftabun Nahar*, Sumaia Kashem, Anutosh Das \\ Department of Urban \& Regional Planning, Rajshahi University of Engineering and Technology, Rajshahi, Bangladesh \\ Email address: \\ himu2010.13@gmail.com (A. Nahar) \\ ${ }^{*}$ Corresponding author
}

To cite this article:

Aftabun Nahar, Sumaia Kashem, Anutosh Das. Regionalization of Bangladesh: A Key to Identify Regional Disparities of the Pattern of Population Distribution. Landscape Architecture and Regional Planning. Vol. 4, No. 1, 2019, pp. 5-9. doi: 10.11648/j.larp.20190401.12

Received: December 22, 2018; Accepted: January 28, 2019; Published: February 27, 2019

\begin{abstract}
Bangladesh is a populous country in the world. Nowadays, urbanization takes the form of rapid growth of urban population. As a result, population density is being increased that influences average household size. This paper attempts to understand the key issues related to the population distribution. The purpose of the study is delineation of the formal region based on the population density, growth rate and average household size in context of sixty four districts of Bangladesh. The data are collected from Bangladesh Bureau of Statistics (BBS), 2011. It analyzes the status of the regions which are fixed by Composite Index method. Among three classification methods, Mean-SD method is used to classify the region. Because, more symmetrical shape of histogram of normal distribution is found in Mean-SD method. It arranges the distribution of population within the 64 districts from high populated to low populated region which represents the actual population scenario of Bangladesh such as Dhaka, Narayanganj are high populated and Rangamati, Khagrachari, Barguna, Pirojpur are in the low populated region. This study gives a scope for future development of region and will be helpful for provision of facilities according to the ranking.
\end{abstract}

Keywords: Formal Region, Population Density, Growth Rate, Average Household Size, Regionalization

\section{Introduction}

Bangladesh has the highest population density in the world. According to the World Bank, population in Bangladesh is increased from 1990 to 2008 with 44 million, which indicates $38 \%$ growth in population [1]. With 152.51 million people living in 147,5700 square kilometer area, the significance of population in the context of Bangladesh cannot be under-emphasized [2]. The development effort of the country is therefore critically dependent on controlling the growth of population and efficiently managing this huge mass [3]. However, it is surprising that in the most densely populated country in the world, the population problem has not been considered with due importance. The well-being of people can be affected by the policies regarding population growth. Moreover, the average household size is another factor that affects the standard living of the people. The previous researches are mainly based on population problems and policies, rapid growth of urbanization, spatial characteristics considering only one or two factors of population. In Population Monograph of Bangladesh, distribution of population is shown according to division, sex and rural-urban residence. [4]. But those researches are not concerned about formal regionalization based on factors of population. In this study, we try to delineate formal region in Bangladesh on the basis of three variables such as growth rate, population density and household size by using Composite Index Method. However, the combined effects of these three variables are considered to delineate the major three regions of Bangladesh. Population density is very high in Bangladesh. So, population distribution should be known to provide any kind of facilities and emergency relief for affected peoples. It is necessary to fulfill people's demand and ensures social justice.

\section{Literature Review}

This section of this paper illustrates the basic concepts of regionalization and related researches. According to Glasson, 
the concept of the region as a method of classification has evolved through two distinct phases reflecting the economic advance from a simple agrarian economy to a complex industrial system. The first phase involves the concept of the formal region that is concerned with uniformity and defined according to homogeneity. Where the second phase concerning with interdependence called functional region [5]. Formal region delineation is the main objective of this study. To delineate formal regions, local units having similar characteristics are being grouped together according certain similar criteria [6] Earlier, the criteria were based on physical characteristics such as topography, climate or vegetation linked with the concept of geographical determinism. Later there was shift to the use of economic, social and political criteria. The criteria is being used on the basis of data availability. Formal regions can be identified on the basis of homogeneity, modality or polarization, a programming or policy etc. [7]. In this study, formal regions are delineated based on population distribution pattern and the criteria are the population density, growth rate and average household size.

In previous researches, formal regions are delineated in Bangladesh based on different criteria. Bhuiya \& Mohiuddin has conducted a research on formal region delineation within Bangladesh on the basis of agricultural productivity. The low productive region normally depends on high productive region. The study include this spatial dependencies among the districts. To delineate the formal region, Productivity Index method has been used. It is widely used method to calculate individual and composite productivity condition. The districts has been divided into four regions on the basis of combined productivity index and composite score. The high the productivity index the high productive region it is. The percentage of moderately high productive region $(34.2 \%)$ is more than the others [8]. The study also uses Moran-I concept for the identification of spatial dependencies among the districts and reached a conclusion that about 49 districts are not in significant dependencies. To identify priority of development, this study has a greater significance.

Another Study was conducted by Rahman on formal region delineation that examines various aspects of urbanization and spatial inequalities in economic development of Bangladesh. The delineation is done based on three variable population density, degree of urbanization and environment quality. Factor and cluster analysis method has been used for this study on the basis of BBS data, 1991. The study reached at a conclusion that three metropolitan districts; Dhaka, Chittagong, and Khulna are relatively too large and contiguous. Only few districts are homogeneous between less developed districts. Some contiguous and compact districts have either enclaves or outliners such as Narayanganj, Gazipur [9]. Findings of the analysis are presented in this paper in two ways which would be helpful for future development planning. It divided the districts into more manageable homogeneous groups as a focus for further study and it provided opportunities for detail observation into the nature and changes in urbanization and spatial development in each individual district.

\section{Methodology}

The principal objective of this study is to find out some formal region within Bangladesh based on the population distribution as stated in introduction. For identifying this distribution, population growth rate, population density and average size of household are important factors. With the increase of population in any area, the number of people living per kilometer area that means population density automatically arises as the area being fixed. And hence the average size of household which represents the number of person living into one room also increases. The value of Pearson Correlation is closer to +1 indicate the strong positive linear relationship among variables. Here the positive correlation among the factors helps us for the selection of variables (table 1).

Table 1. Correlations among Selected Variables.

\begin{tabular}{llll}
\hline Correlations among Selected Variables & growth_rate_X1 & Average household size_X2 & Population Density_X3 \\
\hline growth_rate_X1 & Pearson Correlation & 1 & .080 \\
Average household size_X2 & Pearson Correlation & .080 & 1 \\
Population Density_X3 & Pearson Correlation & $.401^{* *}$ & .138 \\
\hline
\end{tabular}

Source: Prepared by authors.

In case of possessing of two or more variables, composite index is used [10]. Again the selected variables are in same direction so the primary objective involves this analytical approach. As Based on the availability of data in Bangladesh, the data used in this study is collected in the forms of units, an administrative district. The present study includes 64 administrative districts of Bangladesh. Most government census data from Bangladesh Bureau of Statistics, 2011 was available.

To make the data comparable, prior to any data aggregation, normalization is required as the indicators doesn't have same measuring unit. The study has selected three variables having different measuring unit. Therefore for the necessity of bringing those into same standard, those are being transformed into dimensionless number by calculating logarithm of the dataset of three variables. The ratio of mean and standard deviation for each variable refers the respective weight of the three variables $\left(\mathrm{W}_{1}, \mathrm{~W}_{2}, \mathrm{~W}_{3}\right)$. The formula of calculating combining weight of three variables is given below-

$$
\mathrm{W}=\left\{\left(\log \mathrm{X}_{1} \times \mathrm{W}_{1}\right)+\left(\log \mathrm{X}_{2} \times \mathrm{W}_{2}\right)+\left(\log \mathrm{X}_{3} \times \mathrm{W}_{3}\right)\right\} \div\left(\mathrm{W}_{1}+\mathrm{W}_{2}+\mathrm{W}_{3}\right)
$$


Using the equation, the composite weight of 64 districts are calculated separately. The next phase involves the phase of reliability checking of ' $\mathrm{W}$ ' through the three methods; Standard Error of Mean, Level of Skewness and Kurtosis.

Table 2. Statistics of $W$

\begin{tabular}{llll}
\hline Statistics of W & & & \\
\hline Mean & 2.01573237 & Skewness & -.197 \\
Std. Error of Mean & .026741246 & kurtosis & 4.141 \\
\hline
\end{tabular}

Source: Prepared by Authors.

From the table 2, the standard error of mean is found .027 that is less than 1 , so the deviation of sample mean is very little comparing with actual mean. And the skewness is 0.197 that refers a negative skewed distribution. As skewness measures the direction and relative magnitude of how far a distribution deviates from normal, the value shows a slight deviation in the left direction and the mass of distribution is concentrated to the right. The value of Kurtosis (4.141) refers that the distribution is leptokurtic that produces more outliers than normal distribution.

The next phase is to classify the data of composite weight. The formula of determining required number of class $\mathrm{k}$ is

$$
2^{\mathrm{k}}=\mathrm{N}[\text { where } \mathrm{N}=64]
$$

That indicates that six classes are required.

Three classification methods are being used, 1) Equal Class Interval method, 2) Mean-SD method, and 3) Arithmetic method. In equal class interval method, the class interval or class size can be determined as

$\mathrm{X}=($ Highest value - lowest value $) \div($ No. of class required $)$

The frequency distribution is shown in table 3.

Table 3. Frequency Distribution of $W$ in equal class interval method.

\begin{tabular}{lll}
\hline Class interval & Region & Frequency \\
\hline $1.279-1.52$ & 1.00 & 2 \\
$1.52-1.76$ & 2.00 & 4 \\
$1.76-2.0$ & 3.00 & 18 \\
$2.0-2.25$ & 4.00 & 36 \\
$2.25-2.49$ & 5.00 & 2 \\
$2.49-2.73$ & 6.00 & 2 \\
\hline
\end{tabular}

Source: Prepared by authors.
In Mean-SD method, the classes are calculated as,

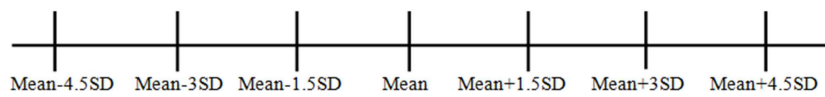

Where Mean $=2.02$ and $\mathrm{SD}=0.21$

The frequency distribution is shown in table 4 .

Table 4. Frequency Distribution of W in Mean-SD method.

\begin{tabular}{lll}
\hline Class & Region & Frequency \\
\hline $1.07-1.39$ & 1 & 1 \\
$1.39-1.7$ & 2 & 3 \\
$1.7-2.02$ & 3 & 26 \\
$2.02-2.33$ & 4 & 32 \\
$2.33-2.65$ & 5 & 1 \\
$2.65-2.96$ & 6 & 1 \\
\hline
\end{tabular}

Source: Prepared by authors.

In Arithmetic method,

$$
\mathrm{A}+\mathrm{X}+2 \mathrm{X}+\ldots \ldots \ldots+\mathrm{KX}=\mathrm{B}
$$

Where, $\mathrm{A}=$ lowest value

$\mathrm{X}=$ class interval

$\mathrm{K}=$ No. of Class

$\mathrm{B}=$ Highest value.

The class interval are calculated as

$[\mathrm{A}-(\mathrm{A}+\mathrm{X})],[(\mathrm{A}+\mathrm{X})-(\mathrm{A}+\mathrm{X}+2 \mathrm{X})],[(\mathrm{A}+3 \mathrm{X})-(\mathrm{A}+3 \mathrm{X}+3 \mathrm{X})] \ldots$ The frequency distribution is shown in table 5 .

Table 5. Frequency Distribution of $W$ in Arithmetic Mean method.

\begin{tabular}{lll}
\hline Class & Region & Frequency \\
\hline $1.279-1.349$ & 1.00 & 1 \\
$1.349-1.487$ & 2.00 & 1 \\
$1.487-1.694$ & 3.00 & 2 \\
$1.694-1.974$ & 4.00 & 12 \\
$1.974-2.315$ & 5.00 & 46 \\
$2.315-2.734$ & 6.00 & 2 \\
\hline
\end{tabular}

Source: Prepared by authors.

Selection of method is one of the important tasks to finalize the class interval with more accuracy. Three histograms are given below together (figure $1 \& 2$ ) to make better comparison.

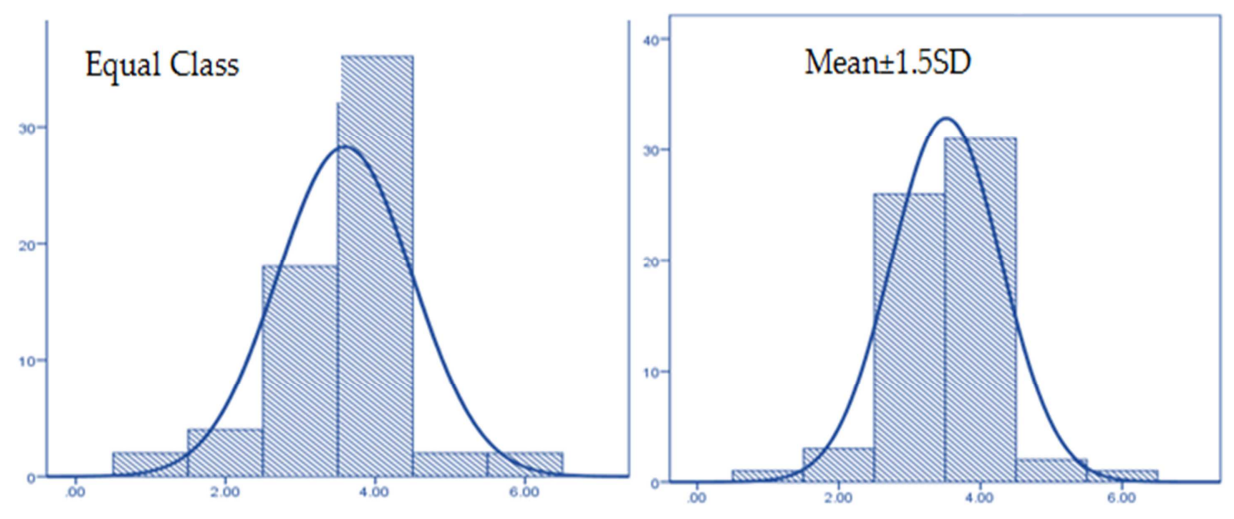

Source: Prepared by Authors, 2018.

Figure 1. Histograms of Equal Class Interval Method and Mean $\pm 1.5 S D$ Method. 


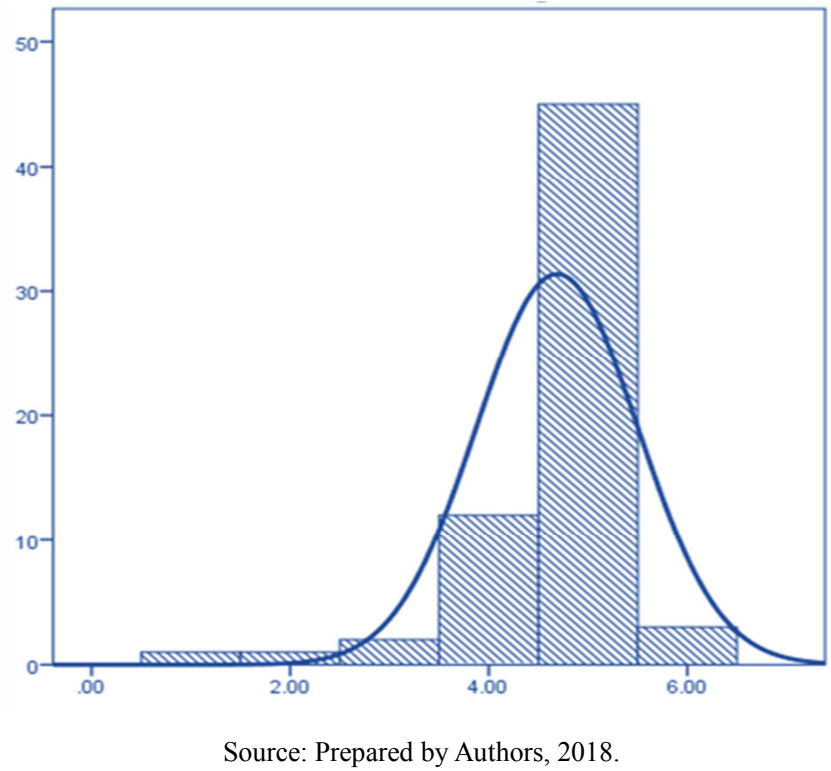

Figure 2. Histograms of Arithmetic Mean Method.

In this study, Mean $\pm 1.5 \mathrm{SD}$ method is selected comparing the histograms because of having more symmetrical shape comparing the others. In equal class interval method, the presence of large peak at one tail deviates it from normal distribution. Again in Arithmetic mean method, the distribution is left-skewed distribution having greater mass of distribution on right side.

According the class interval, 64 districts are grouped into 6 regions. The chronological sequence of region 1 to 6 represents the order of weight (low to high). That means region 1 has the lowest distribution of population where region 6 has the maximum distribution of population.

For the favour of analysis, the six regions has been regrouped into three main region; Highly populated region, Medium populated region and low populated region.

\section{Data Interpretation and Result}

From the Methodology it is seen that the 64 districts are assigned to three groups; Highly populated region, Medium populated region and Low populated region (shown in Figure 3).

The medium region covers the maximum districts of Bangladesh. That refers the growth rate, average household size and population density are in average range of ranking which is medium range.

The low populated region comprises of only four districts such as Rangamati, Khagrachari, Barguna, Pirojpur. The geographical characteristics can be included as one of the important reason for the low concentration of population here.

For example, Rangamati and Khagrachari are hilly area of Bangladesh situated at Chittagong division. The largest city Rangamati has an area of 6116 square kilometer of which 4825 square kilometer are under forest and 1296 square kilometer are riverine area [11]. Again Khagrachari district is a valley with three rivers. The two area cover the largest portion of extensive hilly area of Bangaldesh, Chittagong Hill Tracts [12].
On the other hand, the districts Barguna is the coastal area where the maximum area are riverine [13]. And most of riverine area is in Pirojpur also [14]. The four districts mainly depends on agriculture so the outer migration rate is higher in those districts for better sources of work which is tough there due to vulnerable geographic conditions.

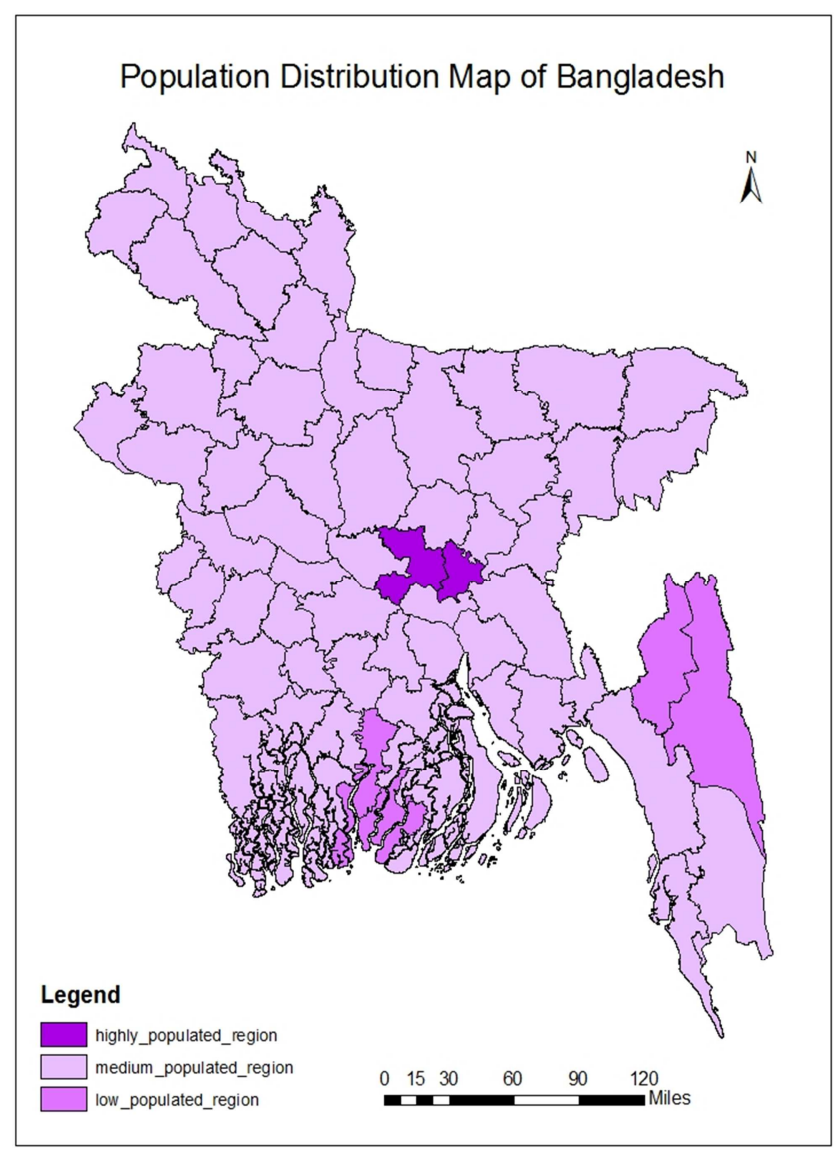

Source: Prepared by Authors, 2018.

Figure 3. Population Distribution Map of Bangladesh.

Moreover, the highly populated region only consists of two districts; Dhaka, Narayanganj. The inner migration increases population density and average household size in these districts [15]. For example, Dhaka is capital of Bangladesh having the maximum no of sources of working. According to Terry, the population of Dhaka is growing by an estimated $4.2 \%$ per year, one of the highest rate amongst Asian cities. The continuing growth reflects the ongoing migration from rural areas to the Dhaka urban area which accounted $60 \%$ of city's growth in 1980s [16]. Rural peoples gather into Dhaka for source of work as well as a little bit higher standard for living. That results excessive population density.

The second high populated district Narayanganj has 5266 population per square kilometer with population growth rate 3.05 and average household size 4.34 . The cottage industry like weaving and cotton is abundant here [17]. International trading, import and export business, shipyard brickfield etc. create employment opportunities to people that attracts inner migration in this district and results higher population. 


\section{Conclusion}

The study examined the spatial nature of population and spatial development in Bangladesh. Composite weighted index method is used to identify the distribution of population. This is nothing but a sort of general methodology that can be used for future studies of any country about population distribution. This study divided the districts into homogeneous groups as a focus for further study. It provided opportunities for detail observation into the nature and changes in population in each individual district. Similarities and differences between and among the districts in a region provide better understanding of the problems of population and thus would be helpful for future regional development planning.

Analysis of simple graphical mapping of factor scores is useful in that it describes the spatial (inter-district) patterns of variation. Medium regions are more in number in Bangladesh. Dhaka and Narayanganj are high populated due to increased number of migration. Migration increases population density and average household size that makes the district under developed. And Khagrachari \& Rangamati are in low populated region due to outer migration and vulnerable geography. The study recommends decentralization of facilities from highly populated zone to medium and low populated zones, so that there is a balance of daily living facilities in everywhere of Bangladesh.

\section{References}

[1] Demographics of Bangladesh Wikipedia. Retrieved September 15,2017 , from https://en.m.wikipedia.org/wiki/Demographics_of_Banglades h.

[2] Population and Housing Census (2011). Socio-Economic and Demographic Report: Bangladesh Bureau of Statistics (BBS), Statistics and Informatics Division (SID), Ministry of Planning, Government of the People's Republic of Bangladesh.

[3] Farid, K. S., Ahmed, J. U., Sarma, P. K. \& Begum, S. (2011). Population Dynamics in Bangladesh: Data sources, current facts and past trends. Journal of Bangladesh Agricultural University, 9 (1), pp. 121-130, ISSN: 1810-3030.

[4] Population Monograph of Bangladesh (2015). Population Distribution and Internal Migration in Bangladesh:
Bangladesh Bureau of Statistics (BBS), Statistics and Informatics Division (SID), Ministry of Planning, Government of the People's Republic of Bangladesh. ISBN978-984-33-9951-9.

[5] Glasson, J. (1974). An introduction to regional planning. Hutchinson \& Co (Publishers) Ltd Fitzroy Square, London.

[6] Delineation of Formal Region: Planning Techniques. Retrieved September 15, 2017, from https://planningtank.com/planning-techniques/delineation-offormal-regions

[7] Methods of Regionalisation for Identification of Formal Regions. Retrieved September 15, 2017, from http: //www.yourarticlelibrary.com/geography/methods-ofregionalisation-for-identification-of-formal-regions/4223.

[8] Bhuiya, M. M. R. \& Mohiuddin, H. (2013). Agricultural Regionalization of Bangladesh Based on Productivity and Analysis of Spatial Dependencies of for Productivity between the Districts of Bangladesh. Journal of Bangladesh Institute of Planners, 6, pp. 181-189.

[9] Rahman, M. M. (2004). Regionalization of Urbanization and Spatial Development: Planning Regions in Bangladesh. The Journal of Geo-Environment, 4, pp. 31-46.

[10] Bo, C. \& Pau, W. Y. (2008). A Composite Index of Economic Integration in the Asia-Pacific Region, Pacific Economic Cooperation Council's State of the Region Project.

[11] Rangamati District Wikipedia. Retrieved September 15, 2017, from https://en.wikipedia.org/wiki/Rangamati.

[12] Khagrachari District Wikipedia. Retrieved September 15, 2017, from https://en.wikipedia.org/wiki/Khagrachari_District.

[13] Barguna District Wikipedia. Retrieved September 15, 2017, from https://en.wikipedia.org/wiki/Barguna.

[14] Pirojpur District Wikipedia. Retrieved September 15, 2017, from https://en.wikipedia.org/wiki/Pirojpur_District.

[15] Dhaka Wikipedia. Retrieved September 15, 2017, from https://en.wikipedia.org/wiki/Dhaka.

[16] Terry, M. (2001). Urbanization Takes on New Dimensions in Asia's Population Giants: Population Reference Bureau. Retrieved September 15, 2017, from https://www.prb.org/urbanizationtakesonnewdimensionsinasia spopulationgiants/.

[17] Narayanganj District Wikipedia. Retrieved September 15, 2017, from https://en.wikipedia.org/wiki/Narayanganj. 